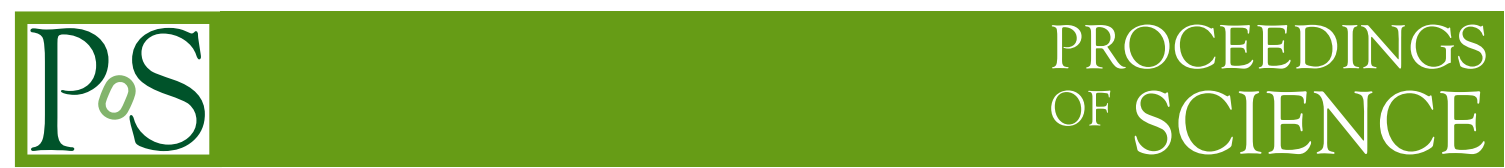

\title{
Status of Water Cherenkov Detector Array of LHAASO project
}

\section{Mingjun chen ${ }^{1}$ on behalf of LHAASO colloboration}

Institute of High Energy Physics, Chinese Academy of Scienes

No.19-B, Rd Yuquan, Beijing, China

E-mail:mjchen@ihep.ac.cn

The Water Cherenkov Detector Array (WCDA) is an important component of Large High Altitude Air Shower Observatory (LHAASO), which mainly aims at surveying the northern sky for VHE gamma ray sources. The WCDA has an area of $78,000 \mathrm{~m}^{2}$, and is sub-divided into 3,120 cells by black curtains. Currently, the $\mathrm{R} \& \mathrm{D}$ is finished. In this paper, the basic design, performance and status of WCDA will be introduced.

35th International Cosmic Ray Conference - ICRC2017

10-20 July, 2017

Bexco, Busan, Korea

\section{${ }^{1}$ Speaker}




\section{Introduction}

The Large High Altitude Air Shower Observatory (LHAASO) project[1,2] is a new generation multi-component instrument, and under construction at 4,410 meters of altitude in the Sichuan province of China, with the aim to study with unprecedented sensitivity the spectrum, the composition and the anisotropy of cosmic rays in the energy range between $10^{12}$ and $10^{18} \mathrm{eV}$, as well as to act simultaneously as a wide aperture (one stereoradiant), continuously-operated gamma ray telescope in the energy range between $10^{11}$ and $10^{15} \mathrm{eV}$. Very-high-energy (VHE, $>100 \mathrm{GeV}$ ) gamma ray astronomy has been an interesting field over the past 30 years. Since the first detection of $\mathrm{TeV}$ gamma rays from the Crab Nebula with the Whipple experiment in 1989[3], more than 100 sources of VHE gamma ray emission have been discovered. Among these sources, more than 60 have been identified as galactic, including supernova remnants, pulsar wind nebulae, and gamma ray binaries; the rest, except the unidentified ones, have been of extragalactic origin. Surveying the sky for more sources in this energy band and monitoring their emission intensity are very important to understand the evolution of galaxies and the acceleration mechanisms in gamma ray sources.

As one of the major components of LHAASO, Water Cherenkov Detector Array(WCDA) $[4,5]$ using the Cherenkov radiation mechanism via the water media, has very high duty-circle $(>95 \%)$, and large field of view $(>2 \pi / 3)$, and great background rejection power. Gamma rays and cosmic rays enter the Earth at the top of the atmosphere, interacting with the air nuclei to form air shower. The secondary particles in the air shower, such as gamma photons, positron and electrons, muon and a few hadrons, will reach the ground and hit the WCDA detector to produce Cherenkov light in the water. Part of the Cherenkov light will be collected by a large area of the photomultiplier tube, converted into electrical signals, transfered to the the electronics system, and ultimately formated digital signal data. These digital signals have information such as the arrival time of the secondary particles, the charge of generated signal. Combined with the triggered detectors unit position, with the data reconstruction, the direction and other parameters relatiing with primary cosmic ray particles, can be determined.

\section{Detector}

The WCDA covering an area of about $78,000 \mathrm{~m}^{2}$ area, is constituted by 3,120 detector units divided into 3 separate arrays (Fig.1). Every array is a single water pond with a $4.4 \mathrm{~m}$ depth. Two of them with an effective area of $150 \times 150 \mathrm{~m}^{2}$ contain 900 detector units each. The third pond with an area of $300 \times 110 \mathrm{~m}^{2}$ contains 1,320 detector units. Each detector unit has a $5 \times 5 \mathrm{~m}^{2}$ area and is divided by black plastic curtains vertically hung in the water to attenuate scattered light. The curtains of the cells are made in black plastic to minimize late light from reflections. A single 8/9" PMT in each unit is deployed at the center of the cell bottom. To guarantee an attenuation length of near-ultra-violet light longer than 15 meters a water purification system will be operated. 




Figure 1, the layout of WCDA.

According to the experimental requirements, WCDA pool (Figure 2) must be a strict dark, impermeable, sealed, insulation system. The roof is made of light steel structure and sandwich panel, in which the steel frame structure is strictly rust-proof. Pool side wall with reinforced concrete pouring, the bottom of the pool for the sand, non-woven and other cushion, the pool is distributed within the column structure for supporting the roof. The side wall of the pool and the bottom of the lining with $2 \mathrm{~mm}$ thickness HDPE film to ensure that the experimental water from the outside world, and the daily leakage rate less than $0.1 \%$. In the pool, in order to avoid the light crosstalk between the unit detectors, the unit detectors are separated by a curtain (size $3.3 \mathrm{~m} \times 4.7$ $\mathrm{m}$ ), and the material is made of a black HDPE film. The curtain system is hung through a stainless steel pipe network, and the stainless steel pipe network is hung on the roof structure by wire. In order to avoid uneven ground subsidence, which may cause the location of the photomultiplier tube movement, the photomultiplier tubes is also suspended above the stainless steel pipe network. Each PMT requires detailed performance testing and waterproof packaging. And the electronic boards are located in the pool of distributed electronic boxes. During the operation of the detector, the realtime time calibration, the charge calibration and water quality measurement, and using the slow control system to monitor the pool environment will be applied. In order to meet the needs of the detector for the extended dynamic range measurements in the field of cosmic rays, an additional dynamic range extended system will be deployed in one of the ponds [6].



Figure 2, the schematic of basic structure of the pond. 


\subsection{Photomultiplier Tube}

Most of the energy spectrum of gamma ray radiation source is presented as the exponential distribution with the energy increase sharply. Meanwhile, the Cherenkov light signal generated by the secondary gamma ray of the air shower in the water, is received by the photomultiplier tube, which about $40 \%$ are single photoelectrons. Thus it' mandatory for PMT good single PE measurement. The energy of the secondary particles is also close to the distribution of the exponential, and the density depends on the distance from the shower core, and the proportion of the large signal is quite low. For example, more than 4,000 photoelectrons account for only 1/10,000 or less of all the signals. Therefore, we chose 4,000 photonelectrons as the upper limit of the charge measurement of the large area photomultiplier tube with help of the design of the base using one dynode output. In the meantime, the angular resolution depends on the time measurement. Generally, the transit time spread of PMT is the most important parameter which is quite related to the precision of time measurement, and should be less than 3ns typically.

In addition, WCDA also requires PMT has a little lower noise rate, and small after pulse ratio. In order to reduce the cost of HV system, three PMTs will be powered by one HV channel, therefore, the uniformity of HV work values should be reasonable. Finally, there are two types of PMTs which satisfy the WCDA's requirements after a long time collaboration with some manufactories. One is CR365 from Beijing Hamamatsu, and the other is XP1805 from HZC Photonics [7]. Coincidently, these two types of PMTs are both produced in China. CR365 has better P/V ratio, time resolution and quality control, On the other hand, XP1805 has better linearity, smaller after pulse ratio, etc. WCDA will choose one type after the contract negotiation in this August.

\subsection{Water Quality Control}

It is always essential for water Cherenkov detectors to keep the quality of water for the whole operating period. A water purification and recirculation system will be deployed for this purpose. The water purification are made up of the following components: a multimedia filter, an activated carbon filter, two fine filters $(5 \mu \mathrm{m}$ and 1 $\mu \mathrm{m})$, an ultra-fine filter $(0.22 \mu \mathrm{m})$, and UV lamps (254 $\mathrm{nm}$ and $185 \mathrm{~nm})$. The UV lamp at $185 \mathrm{~nm}$ is especially important for the system because it could destroy the dissolved total organic carbon (TOC) which is believed to the main source of the pollution in the water. The water purified by this system can easily reach the transparency (attenuation length) above $15 \mathrm{~m}$ for lights at around $400 \mathrm{~nm}$ wavelength, fulfilling the requirement of the experiment. Water from a sedimentation pond is supplied to the purification system, and then poured by pumps into the pond through a pipe net distributed at the bottom of the pond. Many small holes are punched along these pipes, allowing a uniform injection of the water. Another similar set of pipe net is installed on the top of the pond, about $40 \mathrm{~cm}$ below the water surface, starting to continuously draw the pond water to the purification system after the pond has been filled. And the flow of recirculation system is one volume/month.

\subsection{Electronics}

In order to accurately record the arrival time and the charge of the PMT signal, the detector's requirements for electronics system[8] are as follows:

1) Dynamic range: S.P.E. (Single Photoelectron) 4,000 PE. 
2) Charge resolution accuracy: <30\% @ S.PE, <3\% @ 4,000 PE.

3) Time resolution accuracy: $<500$ ps RMS.

4) time measurement resolution (bin size): <1 ns.

5) time measurement dynamic range: $>2 \mu \mathrm{s}$.

6) single channel average count rate: $\sim 50 \mathrm{kHz}$.

The PMT signal amplitude would vary from 1 PE to 4,000 PE. while the discrimination threshold is as low as 0.33 PE. By adjusting the gains of the buffers in the two readout channels, the anode channel can effectively cover a range of 1-133 PE. while the dynode channel covers a range of 30-4,000 PE. A total dynamic range of 4,000 is achieved and there exists enough overlap between the two channels. The PMT signal from the anode is also used for time measurement. After passing through the discrimination circuits, the hit signal is digitized by a Time to Digital Converter (TDC) integrated in the FPGA. As for precise charge measurement, we can employ the digital peaking method, in which the PMT is digitized by an ADC after amplification and shaping, then the peak value of the amplitude can be sought based on the digital signal processing.

Since the FEEs are distributed over a large area, the delay difference among the long signal transmission paths would cause non-aligned phases between the clock signals of different FEEs, which cannot be tolerated in high resolution time measurement. Besides, aging of the electronics and temperature variation would lead to fluctuation of the time delay.A novel technology named White Rabbit (WR) has recently been developed in the frame of CERN's renovation projects. It is capable of controlling thousands of nodes over a few kilometers with a good timing accuracy. As for the timing, its kernel technique is based on the Precision Time Protocol (PTP, IEEE1588) and the Synchronous Ethernet technology. Invoked by the WR technique, we employ an adaptive delay measurement and compensation structure[9]. And the clock phase synchronization accuracy is better than $0.3 \mathrm{~ns}$

\subsection{Calibration}

During the operation of the detector, the time calibration and the charge calibration will be proceeded respectively. The time calibration is realized by an optical fiber system. On side top of every PMT, two optical fibers with approximately $40 \mathrm{~m}$ in length are installed. All the fibers in a cluster, which contains $6 \times 6$ cells, are bundled together, illuminated by a uniform light source consisting of an array of LEDs. These LEDs are powered by a same circuit which can guarantee a fast rise-time $(<10 \mathrm{~ns})$ of the LED light pulse output. For every cluster, at least two fibers are exchanged with two neighboring clusters, enabling a cross calibration of clusters. LEDs are pulsed in a rate of $10 \mathrm{~Hz}$, automatically triggering the DAQ system, so that there is enough statistics to calibrate the whole pond in a time scale of one hour, while the data-taking for air showers keeps continuously running. With a proper selection and design of the fibers, LEDs, the drivers, and the cross calibration method, all the PMTs in the pond can achieve a precision $<0.2 \mathrm{~ns}$ for the time offset measurement.

The charge of the PMT signals is calibrated in two ranges: the single photoelectron spectrum of the counting rate and the signal of nearly vertical muons hitting the PMT cathode. For the former, attributed to the high single rate of PMT in water, it is possible to fit the charge distribution, which is peaked at the S.PE position, so that the gain of each PMT is obtained. For the latter [10], thanks to the enhancement of the number of photo-electrons for muons hitting on the cathode, the peak position of these signals can 
be distinctly revealed after half hour running. This method can calibrate the PMT charge in a precision of $2 \%$, involving all the effects, such as the quantum efficiency, the geometrical effect, the collection efficiency, and the electronics.

\subsection{Triggering}

Based on the "triggerless" mode of the overall structure requirements, all data of the front-end electronics need to be read out to the DAQ system for data selection and analysis. The single channel count rate is estimated to be around $50 \mathrm{KHz}[11]$. When the photomultiplier tube is on fire, FEE will record a 128-bit data frame with a data rate of $6.25 \mathrm{Mbps}$, and a total data rate of $24 \mathrm{Gbps}$.

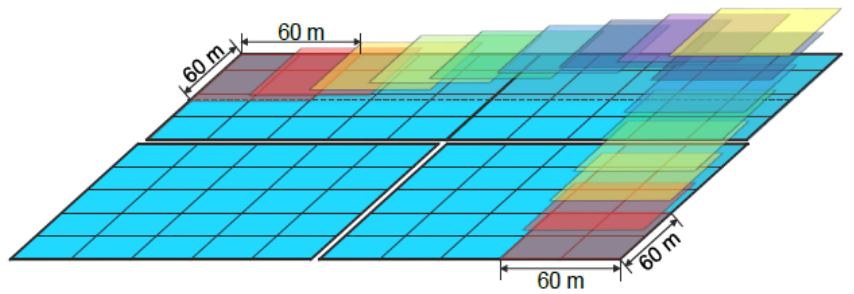

Figure 3, Partition scheme of overlapped trigger clusters.

Taken into account the features of lateral distribution of air showers, and reach a low energy threshold, a natural consideration on the trigger algorithm is to use the multiply zoned pattern. For this purpose, the array is split into 72 approximately halfoverlapped trigger clusters, each containing 144 cells and covering an area of $60 \mathrm{~m} \times 60$ $\mathrm{m}$. The graphical representation of the partition of the trigger clusters is shown in Figure 3. Once the number of fired cells in any of these trigger clusters meets some special requirements, the whole array is then set to be triggered. A simple but rather reasonable requirement of the trigger in a trigger cluster is to use the cell multiplicity, judging whether it exceeds a certain number within a short time window. The time window $250 \mathrm{~ns}$ hereby is adopted, corresponding to the time dispersion of particles of a very inclined shower with Zenith angle $55^{\circ}$.

\section{Performance}

With the aid of simulation studies and cost-effective optimization, the best annual sensitivity of WCDA reaches $1.3 \%$ crab nebula near $2 \mathrm{TeV}$.

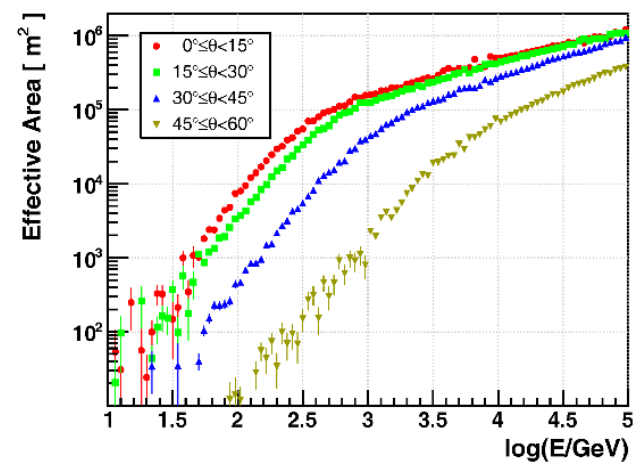

A

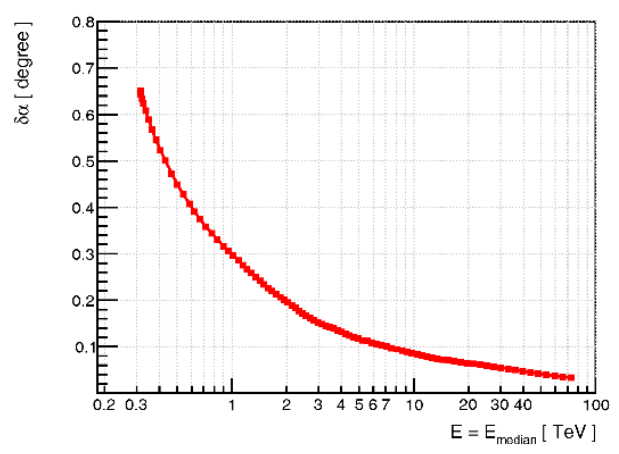

B 


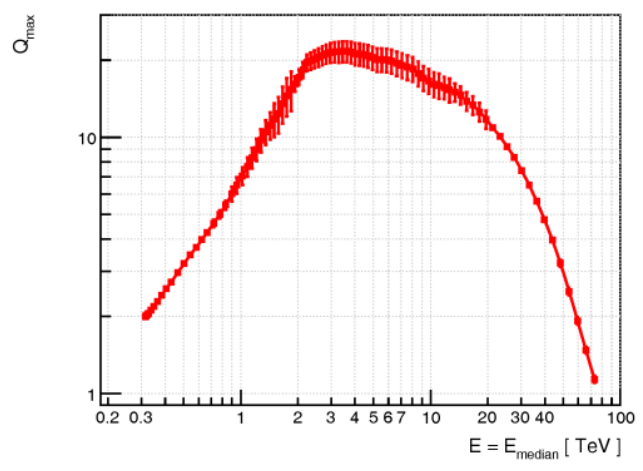

$\mathrm{C}$

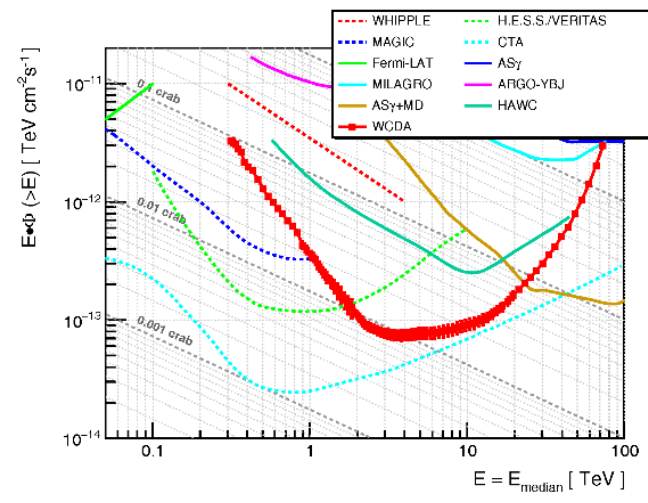

$\mathrm{D}$

Figure 4, plot A shows the effective area of the WCDA as a function of the zenith angle and the energy. Plot B shows the angular resolution of the WCDA. Plot $\mathrm{C}$ shows the $\mathrm{Q}$

factor of proton gamma discrimination capability of the WCDA. Plot D shows the sensitivity of the WCDA.

Figure 4-A shows the effective area of WCDA for gamma rays under different energies. The different points in the figure represent different zenith angles. Figure 4-B shows the angular resolution of the gamma-ray emitted by the CRAB nebular under different energies. The energy in the graph is the median energy with different PMT fire thresholds. Figure 4-C shows the proton gamma discrimination for WCDA under different energies. Figure 4-D is WCDA integral sensitivity as function of the nergies and compared with other experiments. The energy in the graph is the median energy under different PMT fire thresholds. The orbit and energy spectrum of CRAB nebular are used in the calculation.

\section{Summary}

The WCDA in the LHAASO array has a unique advantage, which not only can detect charged particles such as positron, electron, muon, but also photons in the secondary particles of the air shower, because the secondary photon can produce shower or Compton scattering in water. Secondary air shower composition contains a large number of photons (approximately plus or minus 10 times the electrons), so that the detector can put more shower information received, and effectively improve the detection efficiency and accuracy. It also could effectively reject the background, bring a substantial increase in gamma detection sensitivity, and play the site of high altitude advantage, can effectively lower the threshold.

LHAASO project is mainly supported by the Chinese National Development and Reform Commission with more than 1 billon fund, and has already started the capital construction, and the detector installation of WCDA will start in next June, the first pond $(150 \mathrm{~m} \times 150 \mathrm{~m})$ will be operated in the late of 2018 . We hope that the full array of WCDA will start normally run in end of 2020. WCDA of 78,000 $\mathrm{m}^{2}$ water Cherenkov detector array, is nearly four times the area of the latest built in the same category HAWC detector, will play an important role for a long time in the next decade.

\section{Acknowledgments}

This work is supported by National Natural Science Foundation (NSFC) of China under contacts No. 11635011 , No. 11375224 \& 11675187. 


\section{References}

[1] Z. Cao, Chin. Phys. C, A future project at Tibet: the large high altitude air shower observatory (LHAASO), 34 (2): 249-252(2010).

[2] Z. Cao, et al., LHAASO collaboration, Status of the Large High Altitude Air Shower Observatory Project, 33rd ICRC (2013).

[3] T.C. Weekes et al., Observation of TeV gamma rays from the Crab nebula using the atmospheric Cerenkov imaging technique, The Astrophysical Journal, 342:379-395 (1989).

[4] Z.G. Yao, et al. for the LHAASO collaboration, Design and Performance of LHAASO-WCDA experiment, 32nd ICRC (2011).

[5] M.J. Chen et al. R\&D of LHAASO-WCDA. 32nd ICRC (2011).

[6] C. Liu, et al. Dynamic range extended system for the LHAASO-WCDA project, 35th ICRC (2017)

[7] Zhao, X., et al. "Characterization of HZC XP1805 photomultiplier tube for LHAASO-WCDA with a high dynamic range base." Journal of Instrumentation 11.10 (2016).

[8] Zhao, Lei, et al. "Proposal of the Readout Electronics for the WCDA in LHAASO Experiment." Chinese Physics C 38.1 (2013).

[9] C. Li, et al. The clock system for LHAASO WCDA based on reduced White Rabbit Nuclear Science and Techniques, 23:230-236 (2012).

[10] B. Gao, et al, Chin. Phys. C, 38 (2): 026003 (2014)

[11] H.C. Li, et al., Study on single-channel signals of water Cherenkov detector array for the LHAASO project, for the LHAASO collaboration, to appear in Nucl. Instrum.

Methods Phys. Res. A, 854:107-112 (2017) 